\title{
Características dos substratos na absorção de nutrientes e na produção de gérbera de vaso
}

\author{
Fernanda Ludwig'; Dirceu M Fernandes²; Amaralina C Guerrero²; Roberto L Villas Bôas ${ }^{2}$ \\ ${ }^{1}$ UERGS, Av. Independencia 2824, 96815-900 Santa Cruz-RS; ${ }^{2}$ UNESP-FCA, Dep ${ }^{\text {to }}$ Recursos Naturais, C. Postal 237, 18610-307 \\ Botucatu-SP; ludwig.fernanda@yahoo.com.br
}

\section{RESUMO}

O sistema de produção em substratos vem sendo empregado na maioria dos cultivos comerciais de flores e plantas ornamentais, dentre elas, a gérbera. Porém, sua introdução como flor envasada é recente no Brasil e ainda são necessários muitos estudos, especialmente aqueles relacionados com a determinação das propriedades físicas e químicas dos substratos, que contribuam para a sua qualidade produtiva. O presente trabalho foi conduzido com o objetivo de avaliar a influência de características de substratos na absorção de nutrientes e produção de gérbera de vaso. $\mathrm{O}$ experimento foi conduzido em casa de vegetação utilizando o delineamento de blocos ao acaso, esquema fatorial $5 \times 2$ ( 5 substratos e 2 cultivares) e 4 repetições. Os tratamentos consistiram de substratos com diferentes características físicas e químicas e as cultivares utilizadas foram Cherry e Red. As plantas conduzidas em substrato com $\mathrm{pH}$ acima de 7,0 tiveram redução na absorção de ferro, refletindo na menor intensidade de cor verde das folhas. Aquelas conduzidas em substrato com $\mathrm{pH}$ abaixo de 5,0 apresentaram níveis tóxicos de manganês e menor fitomassa seca. As características do substrato, em especial o $\mathrm{pH}$, influenciam a absorção de nutrientes e a produção de gérbera de vaso, podendo alterar a qualidade final da planta.

Palavras-chave: Gerbera jamesonii, plantas envasadas, nutrição de plantas.

\section{ABSTRACT \\ Characteristics of substrates for nutrient absorption and production of gerbera}

The production systems on substrates have been employed in most commercial cultivation of flowers and ornamental plants, including gerbera. However, its introduction as potted flower is recent in Brazil and many studies, especially those related to the determination of substrates physical and chemical properties, which contribute to production quality are still needed. The present study aimed to assess the influence of substrates characteristics for nutrient absorption and production of potted gerbera. The experiment was carried out in greenhouse with the experimental design in randomized blocks, factorial arrangement $5 \times 2$ ( 5 substrates and 2 cultivars) and 4 replications. Treatments consisted of substrates with different physical and chemical characteristics and gerbera cultivars (Cherry and Red). Plants grown on substrate with $\mathrm{pH}$ above 7.0 had a reduction in iron absorption, resulting in lower intensity of green coloration in the leaves. Plants grown on substrate with $\mathrm{pH}$ below 5.0 showed toxic levels of manganese and lower dry mass. The characteristics of the substrate, especially the $\mathrm{pH}$, influence the nutrients absorption and the production of potted gerbera, altering the final plant quality.

Keywords: Gerbera jamesonii, potted plants, plant nutrition.

\section{(Recebido para publicação em 20 de março de 2013; aceito em 17 de abril de 2014) (Received on March 20 2013; accepted on April 17, 2014)}

$\mathrm{O}$ sistema de produção em substratos vem sendo empregado na maioria dos cultivos comerciais de plantas ornamentais, dentre elas a gérbera. De acordo com Guerrero et al. (2012), nos últimos anos o cultivo comercial de gérbera de vaso e de corte tornou-se de grande importância econômica para a floricultura. Porém, sua introdução como flor envasada é recente no Brasil e ainda são necessários muitos estudos, especialmente aqueles relacionados com a determinação das propriedades físicas e químicas dos substratos, que contribuam para a sua qualidade produtiva.

A escolha de um substrato adequado ao cultivo de plantas em vaso requer o prévio conhecimento das suas características químicas e físicas, as quais podem interferir no crescimento de plantas de gérbera (Ludwig et al.,
2010). A limitação do volume para o desenvolvimento radicular exige que o substrato seja capaz de manter água facilmente disponível à planta sem comprometer a concentração de oxigênio no meio (Fermino, 2002), o que é influenciado diretamente pela densidade. Esta expressa a relação entre a massa e o volume do substrato e é importante para a interpretação de outros valores analíticos, como porosidade total, espaço de aeração e disponibilidade de água. Substratos muito leves são indesejáveis pela menor estabilidade do recipiente, ao passo que materiais com elevada densidade tendem a ter menor quantidade de poros ocupados por ar (Bunt, 1984).

$\mathrm{O}$ valor de $\mathrm{pH}$ é uma característica química extremamente importante do substrato, pois relaciona-se sobretudo com a disponibilidade de nutrientes às plantas. Valores inadequados podem afetar o desenvolvimento, principalmente sob acidez excessiva, pois ambientes ácidos têm quantidades menores de nutrientes disponíveis, além das plantas ficarem sujeitas à maior absorção de elementos tóxicos como alumínio e manganês (Fermino, 1996).

A disponibilidade de nutrientes é um dos principais fatores que influenciam a adequação de substratos orgânicos para o crescimento das plantas. Essa disponibilidade pode depender não só da composição do substrato, mas também da capacidade de adsorção, do $\mathrm{pH}$, da estabilidade biológica e da presença de compostos orgânicos dissolvidos (Caballero et al., 2007).

Dessa forma, o presente trabalho foi conduzido com o objetivo de avaliar a influência de características dos substra- 
tos na absorção de nutrientes e produção de gérbera de vaso.

\section{MATERIAL E MÉTODOS}

O experimento foi conduzido em ambiente protegido, na UNESP-FCA, Município de Botucatu-SP. A casa de vegetação possui cobertura em arco com plástico transparente de $150 \mu \mathrm{m}$ de espessura, laterais de tela branca, cortinas de plástico transparente e pavimento com concreto, em uma área total de $168 \mathrm{~m}^{2}$ (7x24 m) e 2,6 m de pé direito. A temperatura média no interior do ambiente durante o período experimental (setembro a novembro de 2008) foi de $21^{\circ} \mathrm{C}$ e a umidade relativa média do ar foi de $55 \%$.

$\mathrm{O}$ delineamento experimental utilizado foi de blocos ao acaso, esquema fatorial $5 \times 2$ ( 5 substratos e 2 cultivares), quatro repetições e 10 plantas por parcela. Os substratos foram selecionados em função de suas características físicas e químicas. As formulações utilizadas foram 1) $40 \%$ terra de subsolo (Latossolo Vermelho Escuro), 40\% casca de pinus, $10 \%$ composição $1\left(\mathrm{C}_{1}\right)(40 \%$ casca de pinus $+30 \%$ vermiculita $+30 \%$ casca de arroz carbonizada), 10\% composição 2 $\left(\mathrm{C}_{2}\right)(75 \%$ casca de pinus $+25 \%$ acícula de pinus); 2) 20\% terra de subsolo, 30\% casca pinus, $30 \% \mathrm{C}_{1}, 20 \% \mathrm{C}_{2}$; 3) $50 \%$ casca de pinus, $20 \%$ fibra de coco granulada, 30\% $\mathrm{C}_{1}$; 4) $30 \%$ casca de pinus, $30 \% \mathrm{C}_{1}, 20 \%$ fibra de coco granulada, $20 \%$ fibra de coco mista; 5) substrato comercial $(70 \%$ casca de pinus $+15 \%$ turfa $+15 \%$ vermiculita). A terra de subsolo ainda é utilizada por produtores com a finalidade de redução de custos, e por isso, optou-se pela sua utilização.

As características químicas de $\mathrm{CE}_{1: 5}$ e $\mathrm{pH}_{1: 5}$ (Brasil, 2007), macronutrientes $_{1: 1,5}$ e micronutrientes 1:1,5 $_{\text {(Sonneveld }}$ \& Elderen, 1994), relação C/N e matéria orgânica (Raij et al., 2001) e físicas de densidade (Brasil, 2007) e retenção de água (de Boodt \& Verdonck, 1972) dos substratos são apresentadas na Tabela 1 .

Mudas de gérbera (Gerbera jamesonii) com quatro folhas definitivas, cultivares Cherry e Red (Dark Eyes) da empresa Sakata Seed Sudamérica (Sakata) foram transplantadas em vasos plásticos pretos, com capacidade para $1 \mathrm{~L}$ (11,5 cm de altura, 13,0 $\mathrm{cm}$ de diâmetro superior e 9,0 cm de diâmetro inferior), preenchidos com o substrato correspondente ao tratamento, de acordo com a densidade úmida. As cultivares selecionadas têm grande aceitação no mercado em função da preferência do consumidor por inflorescências de coloração avermelhada e centro escuro. A cultivar Cherry caracteriza-se por apresentar menor número de inflorescências em relação à 'Red', porém com diâmetro de capítulo superior.

Após o transplante, as mudas foram aclimatadas durante 30 dias, dispostas sobre bancada de madeira $(1,2 \mathrm{~m}$ de largura, 12,0 m de comprimento e 0,8 $\mathrm{m}$ de altura) sob malhas termorefletoras (Aluminet ${ }^{\circledR}$ ) mantendo a intensidade luminosa próxima a 25.000 lux. Após a aclimatação, os vasos foram espaçados de $25 \times 25 \mathrm{~cm}$ e distribuídos em duas bancadas, permanecendo na intensidade luminosa máxima de 50.000 lux, de acordo com Mercurio (2002). A avaliação experimental iniciou após a aclimatação, e os resultados foram apresentados em dias após a aclimatação (DAA).

O manejo da fertirrigação baseou-se na pesagem diária dos vasos, os quais foram mantidos na faixa de 50 a $25 \%$ da água disponível no substrato. A solução nutritiva foi adaptada de Ludwig et al. (2008) e apresentou a seguinte

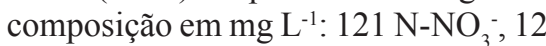
$\mathrm{N}_{-} \mathrm{NH}_{4}^{+}, 92 \mathrm{~K}, 24 \mathrm{P}, 175 \mathrm{Ca}, 27 \mathrm{Mg}$ e $^{+} 9$ $\mathrm{S}, 0,19 \mathrm{~B}, 0,08 \mathrm{Cu}, 2,74 \mathrm{Fe}, 0,19 \mathrm{Mn}$, 0,04 Mo e $0,08 \mathrm{Zn}$ no período vegetativo

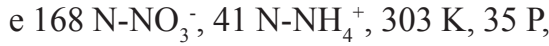
$105 \mathrm{Ca}, 45 \mathrm{Mg}$ e $55 \mathrm{~S}, 0,19 \mathrm{~B}, 0,08 \mathrm{Cu}$, 2,74 Fe, 0,19 Mn, 0,04 Mo e 0,08 Zn no período reprodutivo.

A medidas de intensidade de cor verde (ICV), valor de $\mathrm{pH}$ da solução do substrato e fitomassa seca da parte aérea foram efetuadas ao final do período vegetativo (29 DAA) e ao final do experimento (50 DAA), nas plantas em ponto de comercialização, de acordo com Lin \& French (1985). O teor de nutrientes no tecido vegetal foi quantificado ao final do experimento.

Para a determinação da $\mathrm{ICV}$ da folha utilizou-se o medidor portátil Chlorophyll Meter, modelo SPAD-502 (Soil and Plant Analysis Development) de Minolta Co., Osaka, Japão, cuja unidade é denominada SPAD. A medida foi efetuada no período das 8 às 9 h da manhã, em quatro folhas novas completamente expandidas, por planta. $\mathrm{O}$ valor de $\mathrm{pH}$ da solução drenada do substrato foi aferido pelo método "pourthru" (Cavins et al., 2000).

A parte aérea das plantas foi cortada rente ao substrato, lavada, seca em estufa de ventilação forçada a $65^{\circ} \mathrm{C}$ até fitomassa constante e pesada em balança digital, para determinação da fitomassa seca. Na sequência, as amostras foram moídas em moinho tipo "Willey" e quantificados os teores de nitrogênio $(\mathrm{N})$, fósforo $(\mathrm{P})$, potássio $(\mathrm{K})$, cálcio (Ca), magnésio $(\mathrm{Mg})$, enxofre $(\mathrm{S})$, boro (B), cobre $(\mathrm{Cu})$, ferro $(\mathrm{Fe})$, manganês (Mn) e zinco ( $\mathrm{Zn})$, de acordo com Malavolta et al. (1997).

Os resultados foram submetidos à análise de variância e os efeitos dos substratos e das cultivares tiveram suas médias comparadas pelo teste Tukey a $5 \%$ de probabilidade, quando significativos. Determinou-se, também a correlação de Pearson entre as características avaliadas.

\section{RESULTADOS E DISCUSSÃO}

A intensidade de cor verde (ICV) em folhas de gérbera, o $\mathrm{pH}$ da solução do substrato (Tabela 2), os teores de $\mathrm{N}$, $\mathrm{P}, \mathrm{Mg}, \mathrm{B}, \mathrm{Fe}, \mathrm{Mn}$ e Zn (Tabela 3) variaram significativamente em função dos substratos utilizados. Diferença entre cultivares somente foi observada para os teores de $\mathrm{Cu}, \mathrm{Fe}$, Mn e $\mathrm{Zn}$.

Os menores teores de micronutrientes nas plantas conduzidas no substrato 5 (Tabela 3) relacionam-se diretamente ao pH da solução deste substrato, inicialmente superior a 7,0 (Tabela 1), que tornam micronutrientes como $\mathrm{Fe}, \mathrm{Mn}$ e $\mathrm{Zn}$ menos disponíveis ao longo do ciclo da cultura. Como resultado disso, as folhas novas completamente expandidas apresentaram sintomas de clorose ao qual a gérbera é sensível (Mercurio, 2002), especialmente em solos calcários, devido à insuficiente absorção de Mn ou Fe (Sonneveld \& Voogt, 1997). Em experimento realizado por estes autores, as plantas de gérbera tiveram melhor desenvolvimento em valores de $\mathrm{pH}$ próximos a 5,6.

Avaliando o potencial de diferentes substratos para produção de gérbera em vasos, com especial ênfase no impacto sobre a disponibilidade de nutrientes 
Tabela 1. Características químicas e físicas dos substratos utilizados no experimento (chemical and physical characteristics of substrates used in the experiment). Botucatu, UNESP. 2008.

\begin{tabular}{|c|c|c|c|c|c|}
\hline \multirow{2}{*}{ Características } & \multicolumn{5}{|c|}{ Substratos } \\
\hline & 1 & 2 & 3 & 4 & 5 \\
\hline \multicolumn{6}{|l|}{ Físicas } \\
\hline Porosidade total (\%) & 78,79 & 80,62 & 84,28 & 89,18 & 83,51 \\
\hline Sólidos (\%) & 21,21 & 19,38 & 15,72 & 10,82 & 16,49 \\
\hline Espaço de aeração (\%) & 23,50 & 19,33 & 12,76 & 17,15 & 21,10 \\
\hline Água disponível (\%) & 28,74 & 29,40 & 37,34 & 35,80 & 32,90 \\
\hline Água remanescente (\%) & 26,48 & 31,89 & 34,62 & 36,23 & 29,50 \\
\hline Densidade úmida $\left(\mathrm{kg} / \mathrm{m}^{3}\right)$ & 750 & 850 & 690 & 590 & 530 \\
\hline Densidade seca $\left(\mathrm{kg} / \mathrm{m}^{3}\right)$ & 538 & 540 & 450 & 298 & 340 \\
\hline \multicolumn{6}{|l|}{ Químicas } \\
\hline $\mathrm{CE}_{1: 5}\left(\mathrm{dS} \mathrm{m}^{-1}\right)$ & 0,54 & 0,49 & 0,26 & 0,28 & 0,48 \\
\hline $\mathrm{pH}_{1: 5 \text { (inicial) }}$ & 5,13 & 4,81 & 5,74 & 5,74 & 7,58 \\
\hline $\mathrm{pH}_{1: 5 \text { (final) }}$ & 5,42 & 4,86 & 5,63 & 5,59 & 6,89 \\
\hline $\mathrm{MO}(\%)$ & 19,00 & 28,00 & 38,00 & 33,00 & 34,00 \\
\hline $\mathrm{C} / \mathrm{N}$ & 10,55 & 15,55 & 21,11 & 18,33 & 18,88 \\
\hline $\mathrm{N}^{-\mathrm{NO}_{3}^{-}-1,1,5}(\mathrm{mg} / \mathrm{L})$ & 77,99 & 122,17 & 2,51 & 12,72 & 7,10 \\
\hline $\mathrm{K}_{1: 1,5}(\mathrm{mg} / \mathrm{L})$ & 70,25 & 86,00 & 45,43 & 56,70 & 176,25 \\
\hline $\mathrm{Ca}_{1: 1,5}(\mathrm{mg} / \mathrm{L})$ & 52,40 & 84,98 & 3,57 & 22,83 & 21,63 \\
\hline $\operatorname{Mg}_{1: 1,5}(\mathrm{mg} / \mathrm{L})$ & 50,15 & 57,60 & 12,13 & 24,45 & 12,83 \\
\hline $\mathrm{Cu}_{1: 1,5}(\mathrm{mg} / \mathrm{L})$ & 0,01 & 0,02 & 0,06 & 0,03 & 0,06 \\
\hline $\mathrm{Fe}_{1: 1,5}(\mathrm{mg} / \mathrm{L})$ & 0,38 & 0,62 & 5,57 & 1,76 & 1,83 \\
\hline $\mathrm{Mn}_{1: 1,5}(\mathrm{mg} / \mathrm{L})$ & 0,19 & 2,48 & 0,20 & 0,38 & 0,84 \\
\hline $\mathrm{Zn}_{1 \cdot 1.5}(\mathrm{mg} / \mathrm{L})$ & 0,04 & 0,11 & 0,06 & 0,06 & 0,08 \\
\hline
\end{tabular}

Substratos: 1) 40\% terra de subsolo (Latossolo Vermelho Escuro) $+40 \%$ casca de pinus (CP) + 10\% composição $1\left(\mathrm{C}_{1}\right)(40 \% \mathrm{CP}+30 \%$ vermiculita $(\mathrm{V})+30 \%$ casca de arroz carbonizada) $+10 \%$ composição $2\left(\mathrm{C}_{2}\right)(75 \% \mathrm{CP}+25 \%$ acícula de pinus $\left.) ; 2\right) 20 \% \mathrm{LVE}+30 \% \mathrm{CP}$ $+30 \% \mathrm{C}_{1}+20 \% \mathrm{C}_{2}$; 3) $50 \% \mathrm{CP}+20 \%$ fibra de coco granulada (FCG) $+30 \% \mathrm{C}_{1}$; 4) $30 \% \mathrm{CP}+30 \% \mathrm{C}_{1}+20 \% \mathrm{FCG}+20 \%$ fibra de coco mista; 5) substrato comercial (70\% CP $+15 \%$ turfa $+15 \% \mathrm{~V})$ [Substrates: 1) 40\% subsoil earth (dark red oxisol) $+40 \%$ pinus bark $(\mathrm{CP})+$ $10 \%$ compost $1\left(\mathrm{C}_{1}\right)(40 \% \mathrm{CP}+30 \%$ vermiculite $(\mathrm{V})+30 \%$ carbonized rice husk $)+10 \%$ compost $2\left(\mathrm{C}_{2}\right)(75 \% \mathrm{CP}+25 \%$ pinus leaf $\left.) ; 2\right)$ $\left.20 \% \mathrm{LVE}+30 \% \mathrm{CP}+30 \% \mathrm{C}_{1}+20 \% \mathrm{C}_{2} ; 3\right) 50 \% \mathrm{CP}+20 \%$ granulated coconut fiber $\left.(\mathrm{FCG})+30 \% \mathrm{C}_{1} ; 4\right) 30 \% \mathrm{CP}+30 \% \mathrm{C}+20 \% \mathrm{FCG}$ $+20 \%$ mixed coconut fiber; 5$)$ comercial substrate $(70 \% \mathrm{CP}+15 \%$ turf $+15 \% \mathrm{~V})]$.

para as plantas, Caballero et al. (2009) verificaram que a intensidade de cor verde da folha foi inferior em maiores valores de $\mathrm{pH}$, em função da deficiência de Fe. Resultado semelhante foi observado no presente trabalho, em que o teor de $\mathrm{Fe}$ na folha nova completamente expandida e a intensidade de cor verde apresentaram correlação de $0,55^{*}$. A clorose, devido à deficiência deste micronutriente, é o maior problema afetando plantas cultivadas em substratos com $\mathrm{pH}$ alto, caracterizado pelo amarelecimento das folhas novas, em contraste com o verde frequentemente encontrado nas folhas maduras (Caballero et al., 2007). Jeong et al. (2009) descrevem esses sintomas em plantas de gérbera 'Festival Light Eye Pink', como inicial clorose intervenal em folhas recém-maduras, evoluindo para clorose generalizada em quase toda a planta.

Os valores de $\mathrm{pH}$ da solução drenada foram superiores no substrato 5 e inferiores no substrato 2 (Tabela 2). Esta variação deve-se possivelmente à diferença na aplicação de corretivos de acidez pelas empresas fornecedoras dos substratos, conforme verificado na caracterização inicial (Tabela 1). O pH ideal para gérbera situa-se entre 5,5 e 6,5 (Rogers \& Tjia, 1990), com redução na produção em valores acima de 6,0 (Sonneveld \& Voogt, 1997).

Substratos com valores de $\mathrm{pH}$ extremos, inferior a 5,0 e superior a 7,0 não são favoráveis às plantas de gérbera. $\mathrm{O}$ elevado $\mathrm{pH}$ resultou em menor inten- sidade de cor verde e plantas com tons amarelados nas folhagens, enquanto reduzido $\mathrm{pH}$ tornou menor a fitomassa seca da parte aérea (Figura 1), resultando em plantas mais compactas.

A intensidade de cor verde correlacionou-se negativamente com o pH da solução do substrato (Figura 1A), enquanto a fitomassa seca correlacionou-se positivamente (Figura 1B), esses resultados estão em consonância com os obtidos por Caballero et al. (2009). A menor fitomassa nas plantas conduzidas no substrato 2 (Tabela 2), com menor valor de $\mathrm{pH}$, pode estar relacionado ao teor de $\mathrm{N}$, reduzido nas plantas conduzidas nesse substrato (Tabela 3), já que este nutriente tem relação direta com o crescimento vegetativo (Barbosa et al., 
Tabela 2. Fitomassa seca de parte aérea (FSPA), intensidade de cor verde (ICV) em folhas de gérbera e pH da solução drenada do substrato, em função dos substratos e cultivares [dry matter of aboveground part (FSPA), intensity of green color (ICV) in gerbera leaves and pH of the solution drained from the substrate, depending on substrates and cultivars]. Botucatu, UNESP. 2008.

\begin{tabular}{|c|c|c|c|c|c|c|}
\hline & \multicolumn{2}{|c|}{ FSPA (g) } & \multicolumn{2}{|c|}{ ICV (SPAD) } & \multicolumn{2}{|c|}{ pH } \\
\hline & \multicolumn{2}{|c|}{ Dias após aclimatação } & \multicolumn{2}{|c|}{ Dias após aclimatação } & \multicolumn{2}{|c|}{ Dias após aclimatação } \\
\hline & 29 & 50 & 29 & 50 & 29 & 50 \\
\hline \multicolumn{7}{|c|}{ Substratos } \\
\hline 1 & $1,0 \mathrm{abc}$ & 9,3 & $57,4 \mathrm{a}$ & $61,7 \mathrm{a}$ & $5,14 \mathrm{bc}$ & $5,31 \mathrm{~b}$ \\
\hline 2 & $0,8 \mathrm{bc}$ & 7,4 & $58,7 \mathrm{a}$ & $64,0 \mathrm{a}$ & $4,61 \mathrm{c}$ & $4,77 \mathrm{c}$ \\
\hline 3 & $1,0 \mathrm{ab}$ & 10,7 & $54,4 \mathrm{ab}$ & $61,3 \mathrm{a}$ & $5,51 \mathrm{~b}$ & $5,43 \mathrm{~b}$ \\
\hline 4 & $1,1 \mathrm{a}$ & 13,0 & $54,8 \mathrm{ab}$ & $62,1 \mathrm{a}$ & $5,39 \mathrm{~b}$ & $5,51 \mathrm{~b}$ \\
\hline 5 & $0,7 \mathrm{c}$ & 10,6 & $50,4 \mathrm{~b}$ & $47,2 \mathrm{~b}$ & $6,52 \mathrm{a}$ & $6,27 \mathrm{a}$ \\
\hline \multicolumn{7}{|c|}{ Cultivares } \\
\hline Red & 0,9 & 9,3 & 56,3 & 60,7 & 5,42 & 5,41 \\
\hline Cherry & 01,0 & 11,1 & 54,1 & 57,7 & 5,44 & 5,50 \\
\hline $\mathrm{S}$ & $* *$ & $* *$ & $* *$ & $* *$ & $* *$ & $* *$ \\
\hline $\mathrm{C}$ & ns & $* *$ & $\mathrm{~ns}$ & $\mathrm{~ns}$ & $\mathrm{~ns}$ & ns \\
\hline $\mathrm{S}^{*} \mathrm{C}$ & $\mathrm{ns}$ & $* *$ & $\mathrm{~ns}$ & $\mathrm{~ns}$ & ns & $\mathrm{ns}$ \\
\hline $\mathrm{CV}(\%)$ & 21,04 & 9,30 & 7,84 & 10,52 & 7,67 & 4,10 \\
\hline
\end{tabular}

$\mathrm{S}=$ substrato; $\mathrm{C}=$ cultivar; Médias seguidas pela mesma letra minúscula (substratos) e maiúscula (cultivar) não diferem entre si pelo teste Tukey a 5\%. ns: não significativo; **, *significativo a 1 e 5\%, respectivamente. Substratos: 1= 40\% terra de subsolo (Latossolo Vermelho Escuro $)+40 \%$ casca de pinus $(\mathrm{CP})+10 \%$ composição $1\left(\mathrm{C}_{1}\right)(40 \% \mathrm{CP}+30 \%$ vermiculita $(\mathrm{V})+30 \%$ casca de arroz carbonizada $)+10 \%$ composição $2\left(\mathrm{C}_{2}\right)(75 \% \mathrm{CP}+25 \%$ acícula de pinus $) ; 2=20 \% \mathrm{LVE}+30 \% \mathrm{CP}+30 \% \mathrm{C}_{1}+20 \% \mathrm{C}_{2} ; 3=50 \% \mathrm{CP}+20 \%$ fibra de coco granulada $(\mathrm{FCG})+30 \% \mathrm{C}_{1} ; 4=30 \% \mathrm{CP}+30 \% \mathrm{C}_{1}+20 \% \mathrm{FCG}+20 \%$ fibra de coco mista; $5=$ substrato comercial $(70 \% \mathrm{CP}+15 \%$ turfa $+15 \%$ V) $[D A A=$ days after acclimation; $S=$ substrate; $C$ : cultivar; Means followed by the same letters in the same column, being lowercase to substrates and uppercase to cultivars did not differ by the Tukey's test at 5\%. ns = not significant; **, significant at 1 and $5 \%$, respectively. Substrates: $1=40 \%$ subsoil earth (dark red oxisol) $+40 \%$ pinus bark $(\mathrm{CP})+10 \%$ compost $1\left(\mathrm{C}_{1}\right)(40 \% \mathrm{CP}+30 \%$ vermiculite $(\mathrm{V})+30 \%$ carbonized rice husk $)+10 \%$ compost $2\left(\mathrm{C}_{2}\right)(75 \% \mathrm{CP}+25 \%$ pinus leaf $) ; 2=20 \% \mathrm{LVE}+30 \% \mathrm{CP}+30 \% \mathrm{C}_{1}+20 \% \mathrm{C} ; 3=50 \% \mathrm{CP}+20 \%$ granulated coconut fiber $(\mathrm{FCG})+30 \% \mathrm{C}_{1} ; 4=30 \% \mathrm{CP}+30 \% \mathrm{C}_{1}+20 \% \mathrm{FCG}+20 \%$ mixed coconut fiber; $5=$ commercial substrate $(70 \%$ $\mathrm{CP}+15 \%$ turfa $+15 \% \mathrm{~V})]$.

2009). Os teores de $\mathrm{N}$ estão adequados quanto à recomendação de Mercurio (2002) e Ludwig et al. (2008), exceto para as plantas conduzidas nesse substrato.

Os menores teores de $\mathrm{P}$ foram registrados nas plantas conduzidas nos substratos 1 e 2 (Tabela 3). Apesar dos menores valores, estes estão de acordo com os obtidos por Ludwig et al. (2008), com 2,4 $\mathrm{mg} \mathrm{kg}^{-1}$ para Cherry.

$\mathrm{O}$ teor de $\mathrm{Mg}$ na folha foi inferior nas plantas conduzidas no substrato 5 (Tabela 3). Os sintomas de deficiência de $\mathrm{Mg}$ aparecem em folhas mais velhas com clorose internerval semelhante ao $\mathrm{Fe}$ (Mercurio, 2002) por ser componente da molécula de clorofila (Merhaut, 2007). Teor de $2,9 \mathrm{mg} \mathrm{kg}^{-1}$ para cultivar Cherry foi registrado por Ludwig et al. (2008) e de 4,4 mg kg-1 para cv. Red, por Guerrero (2009). A menor concentração nas plantas conduzidas no substrato 5 deve-se ao efeito antagônico em relação ao K (Merhaut, 2007), superior nas plantas crescidas nesse substrato e a elevada concentração inicial de K no substrato (Tabela 1).

$\mathrm{O}$ teor de B foi significativamente superior nas plantas desenvolvidas nos substratos 3 e 4 (Tabela 3). Teores semelhantes foram obtidos por Ludwig (2007) para cultivar Cherry ( $74 \mathrm{mg} \mathrm{kg}^{-1}$ ) e Guerrero (2009) para cv. Red (32 mg $\left.\mathrm{kg}^{-1}\right)$. $\mathrm{O}$ teor de $\mathrm{Cu}$ foi significativamente superior para a cultivar Red (Tabela 4), com valor pouco inferior ao registrado por Guerrero (2009) de 4,7 mg kg-1. Para cv. Cherry, Ludwig (2007) obteve $5,0 \mathrm{mg} \mathrm{kg}^{-1}$.

As plantas conduzidas no substrato 5 apresentaram menores teores de $\mathrm{Fe}$ no tecido vegetal (Tabela 3 ), considerado como nível de deficiência por Anjaneyulu (2008). Nos demais substratos, foram superiores ao recomendado por Mercurio (2002), de 450 a $500 \mathrm{mg}$ $\mathrm{kg}^{-1}$, Jones Junior et al. (1996), de 50 a $200 \mathrm{mg} \mathrm{kg}^{-1}$ e Anjaneyulu (2008), de 225 a $287 \mathrm{mg} \mathrm{kg}^{-1}$. Os baixos teores de $\mathrm{Fe}$ registrados nas plantas com o substrato 5 podem ter contribuído para o surgimento de sintoma de clorose nas folhas. Segundo Mercurio (2002), a disponibilidade de $\mathrm{Fe}$ ocorre quando o $\mathrm{pH}$ do substrato encontra-se entre 3,0 e 6,5, valor inferior ao obtido nesse substrato. Essa observação pode ser confirmada ao analisar a correlação entre o teor foliar de Fe e o pH da solução do substrato, de $-0,69^{* *}$, e entre o teor de $\mathrm{Fe}$ e a intensidade de cor verde, de $0,55^{*}$. De acordo com Caballero et al. (2007) a gérbera é sensível à falta de Fe.

$\mathrm{O}$ teor de $\mathrm{Mn}$ foi maior no substrato 2, para as duas cultivares (Tabela 3). A característica inicial de reduzido $\mathrm{pH}$ (Tabela 1), mantida ao longo do ciclo, possibilitou a maior disponibilidade desse 
Tabela 3. Teor de nutrientes no tecido vegetal de gérbera, em função dos substratos e cultivares (nutrient content in gerbera plant tissue, depending on substrates and cultivars). Botucatu, UNESP. 2008.

\begin{tabular}{|c|c|c|c|c|c|c|c|c|c|c|c|c|}
\hline \multirow{3}{*}{ Substratos } & \multirow{2}{*}{$\mathbf{N}$} & \multirow{2}{*}{$\mathbf{P}$} & \multirow{2}{*}{$\mathbf{K}$} & \multirow{2}{*}{$\mathbf{C a}$} & \multirow{2}{*}{ Mg } & \multirow{2}{*}{$\mathbf{S}$} & \multirow{2}{*}{ B } & \multirow{2}{*}{$\mathrm{Cu}$} & \multirow{2}{*}{$\mathbf{F e}$} & \multicolumn{2}{|c|}{ Mn } & \multirow{2}{*}{$\mathbf{Z n}$} \\
\hline & & & & & & & & & & Red & Cherry & \\
\hline & \multicolumn{6}{|c|}{$(\mathrm{g} / \mathrm{kg})$} & \multicolumn{6}{|c|}{$(\mathrm{mg} / \mathrm{kg})$} \\
\hline 1 & $26,9 \mathrm{bc}$ & $2,3 \mathrm{c}$ & 40,8 & 13,2 & $5,4 \mathrm{a}$ & 1,6 & $42,9 \mathrm{~b}$ & 4,1 & $626 \mathrm{a}$ & 269 bA & $139 \mathrm{bB}$ & $21 \mathrm{bc}$ \\
\hline 2 & $25,9 \mathrm{c}$ & $2,1 \mathrm{c}$ & 43,4 & 14,1 & $4,9 \mathrm{a}$ & 1,6 & $36,9 \mathrm{~b}$ & 4,0 & 831 a & $650 \mathrm{aA}$ & $357 \mathrm{aB}$ & $33 \mathrm{a}$ \\
\hline 3 & $28,9 \mathrm{ab}$ & $3,4 \mathrm{~b}$ & 45,9 & 13,5 & $5,5 \mathrm{a}$ & 1,6 & $57,0 \mathrm{a}$ & 4,3 & 689 a & 191 bA & $91 \mathrm{bA}$ & $25 \mathrm{~b}$ \\
\hline 4 & $27,3 \mathrm{bc}$ & $3,9 a b$ & 47,1 & 13,9 & $5,3 \mathrm{a}$ & 1,6 & $63,2 \mathrm{a}$ & 4,3 & $771 \mathrm{a}$ & $178 \mathrm{bcA}$ & $117 \mathrm{bA}$ & $26 \mathrm{~b}$ \\
\hline 5 & $29,6 \mathrm{a}$ & $4,6 \mathrm{a}$ & 48,8 & 12,4 & $3,6 \mathrm{~b}$ & 1,6 & $38,6 \mathrm{~b}$ & 4,1 & $123 \mathrm{~b}$ & $35 \mathrm{cA}$ & $64 \mathrm{bA}$ & $17 \mathrm{c}$ \\
\hline \multicolumn{13}{|c|}{ Cultivares } \\
\hline$\overline{\text { Red }}$ & 27,2 & 3,3 & 44,7 & 13,6 & 4,9 & 1,6 & 46,2 & $4,3 \mathrm{~A}$ & $706 \mathrm{~A}$ & & & $26 \mathrm{~A}$ \\
\hline Cherry & 28,2 & 3,3 & 45,7 & 13,2 & 5,1 & 1,6 & 49,3 & $4,0 \mathrm{~B}$ & $510 \mathrm{~B}$ & & & $23 \mathrm{~B}$ \\
\hline$S$ & $* *$ & $* *$ & $\mathrm{~ns}$ & $\mathrm{~ns}$ & $* *$ & $\mathrm{~ns}$ & $* *$ & ns & $* *$ & $* *$ & & $* *$ \\
\hline $\mathrm{C}$ & $\mathrm{ns}$ & $\mathrm{ns}$ & ns & ns & $\mathrm{ns}$ & $\mathrm{ns}$ & ns & $*$ & $* *$ & $* *$ & & $*$ \\
\hline $\mathrm{S} * \mathrm{C}$ & $\mathrm{ns}$ & $\mathrm{ns}$ & $\mathrm{ns}$ & $\mathrm{ns}$ & $\mathrm{ns}$ & $\mathrm{ns}$ & ns & $\mathrm{ns}$ & $\mathrm{ns}$ & $* *$ & & $\mathrm{~ns}$ \\
\hline CV (\%) & 5,65 & 14,52 & 18,63 & 11,42 & 11,68 & 10,44 & 14,93 & 8,55 & 33,44 & 35,73 & & 16,38 \\
\hline
\end{tabular}

$\mathrm{S}=$ substrato; $\mathrm{C}=$ cultivar; Médias seguidas pela mesma letra minúscula (substratos) e maiúscula (cultivar) não diferem entre si pelo teste Tukey a 5\%. ns= não significativo; *** significativo a 1 e 5\%, respectivamente; DAA= dias após aclimatação; Substratos: $1=40 \%$ terra de subsolo [Latossolo Vermelho Escuro (LVE)] + 40\% casca de pinus (CP) $+10 \%$ composição $1\left(\mathrm{C}_{1}\right)(40 \% \mathrm{CP}+30 \%$ vermiculita $(\mathrm{V})+30 \%$ casca de arroz carbonizada $)+10 \%$ composição $2\left(\mathrm{C}_{2}\right)(75 \% \mathrm{CP}+25 \%$ acícula de pinus $) ; 2=20 \% \mathrm{LVE}+30 \% \mathrm{CP}+30 \% \mathrm{C}_{1}+20 \% \mathrm{C}$; $3=50 \% \mathrm{CP}+20 \%$ fibra de coco granulada $(\mathrm{FCG})+30 \% \mathrm{C}_{1} ; 4=30 \% \mathrm{CP}+30 \% \mathrm{C}_{1}+20 \% \mathrm{FCG}+20 \%$ fibra de coco mista; $5=$ substrato comercial $(70 \% \mathrm{CP}+15 \%$ turfa $+15 \% \mathrm{~V})$ [S: substrate. C: cultivar; Means followed by the same letters in the same column, being lowercase to substrates and uppercase to cultivars did not differ by the Tukey's test at $5 \%$. ns $=$ not significant; $* *$, significant at 1 and $5 \%$, respectively. $\mathrm{DAA}=$ days after acclimation; Substrates: $1=40 \%$ subsoil earth [dark red oxisol] $+40 \%$ pinus bark $(\mathrm{CP})+10 \%$ compost $1\left(\mathrm{C}_{1}\right)(40 \% \mathrm{CP}+$ $30 \%$ vermiculite $(\mathrm{V})+30 \%$ carbonized rice husk $)+10 \%$ compost $2\left(\mathrm{C}_{2}\right)(75 \% \mathrm{CP}+25 \%$ pinus leaf $) ; 2=20 \% \mathrm{LVE}+30 \% \mathrm{CP}+30 \% \mathrm{C}+$ $20 \% \mathrm{C}_{2} ; 3=50 \% \mathrm{CP}+20 \%$ granulated coconut fiber $(\mathrm{FCG})+30 \% \mathrm{C}_{1} ; 4=30 \% \mathrm{CP}+30 \% \mathrm{C}_{1}+20 \% \mathrm{FCG}+20 \%$ mixed coconut fiber; $5=$ commercial substrate $(70 \% \mathrm{CP}+15 \%$ turf $+15 \% \mathrm{~V})]$.

nutriente, pois, de acordo com Mercurio (2002), esta ocorre principalmente em valores de $\mathrm{pH}$ de 3,0 a 5,5. A correlação linear negativa de $0,69 * *$ entre o valor de $\mathrm{pH}$ do substrato e o teor de $\mathrm{Mn}$ no tecido vegetal evidencia essa observação. Segundo Cavins et al. (2000), o pH é mais importante que a própria nutrição do substrato, pois afeta principalmente a absorção dos micronutrientes. Valores de $\mathrm{pH}$ abaixo de 5,8 podem aumentar a disponibilidade de Fe e Mn, levando a planta à toxicidade.

Os menores teores de Mn no tecido vegetal das plantas desenvolvidas no substrato 5 enquadram-se nos limites inferiores sugeridos por Mercurio (2002) de 40 a $50 \mathrm{mg} \mathrm{kg}^{-1}$ e Jones Junior et al. (1996) de 40 e $250 \mathrm{mg} \mathrm{kg}^{-1}$, porém Ludwig (2007) constatou para a cultivar Cherry, teores de $85 \mathrm{mg} \mathrm{kg}^{-1}$, indicando que as plantas nesse substrato foram deficientes quanto a esse nutriente. Anjaneyulu (2008) considera como deficiente os teores inferiores a $50 \mathrm{mg} \mathrm{kg}^{-1}$.

Quantidades elevadas de Mn nos tecidos vegetais foram observadas no substrato 2 , de forma mais acentuada para a cultivar Red, muito superiores ao recomendado por autores como Mercurio (2002) e Jones Junior et al. (1996), em que o máximo é de $250 \mathrm{mg} \mathrm{kg}^{-1}$. Para Anjaneyulu (2008), teores superiores a $166 \mathrm{mg} \mathrm{kg}^{-1}$ são considerados excessivos para gérbera. Guerrero (2009) obteve valores de $512 \mathrm{mg} \mathrm{kg}^{-1}$ para cv. Red, com sintomas de toxidez, os quais são descritos por Albano et al. (1996) em plantas de Tagetes erecta e Mercurio (2002) em plantas de gérbera, como pequenas pontuações escuras e necróticas, com tamanho e distribuição irregular pelas folhas. Essa caractecteristica parece ter relação com a cultivar, pois o teor de Mn foi superior para 'Red', onde foram observados mais intensamente os sintomas de toxidez, quando conduzidas em substratos com menor $\mathrm{pH}$.

A absorção de Mn decresce com o aumento no $\mathrm{pH}$ do solo e com a reduzida drenagem do mesmo (Humphries et al., 2007). Os substratos com solo (substratos 1 e 2) apresentaram visual- mente um aumento na compactação ao longo do tempo, em função da irrigação descendente ser realizada manualmente em toda a superficie do substrato. As características iniciais desses substratos revelaram elevado espaço de aeração, o que era conferido pelas partículas de solo de grande tamanho, com característica argilosa. A irrigação e o manuseio do substrato promoveu a desintegração destas partículas ao longo do ciclo de crescimento, o que pode ter reduzido a aeração e aumentado a retenção de água, tornando maior a disponibilidade de $\mathrm{Mn}$, a níveis tóxicos para a planta.

$\mathrm{O}$ teor de $\mathrm{Zn}$ tem sua disponibilidade diminuída constantemente à medida que o pH aumenta (Malavolta et al., 1997), conforme verificado na Tabela 3, com correlação linear de $-0,66^{* *}$. Os teores de $\mathrm{Zn}$ referenciados na literatura para a gérbera são altamente discrepantes. Para Mercurio (2002) a faixa considerada adequada é de 6 a $8 \mathrm{mg} \mathrm{kg}^{-1}$, enquanto para Jones Junior et al. (1996) é de 25 a $200 \mathrm{mg} \mathrm{kg}^{-1}$. Ludwig (2007) obteve teores de $24 \mathrm{mg} \mathrm{kg}^{-1}$ para a cultivar Cherry. 


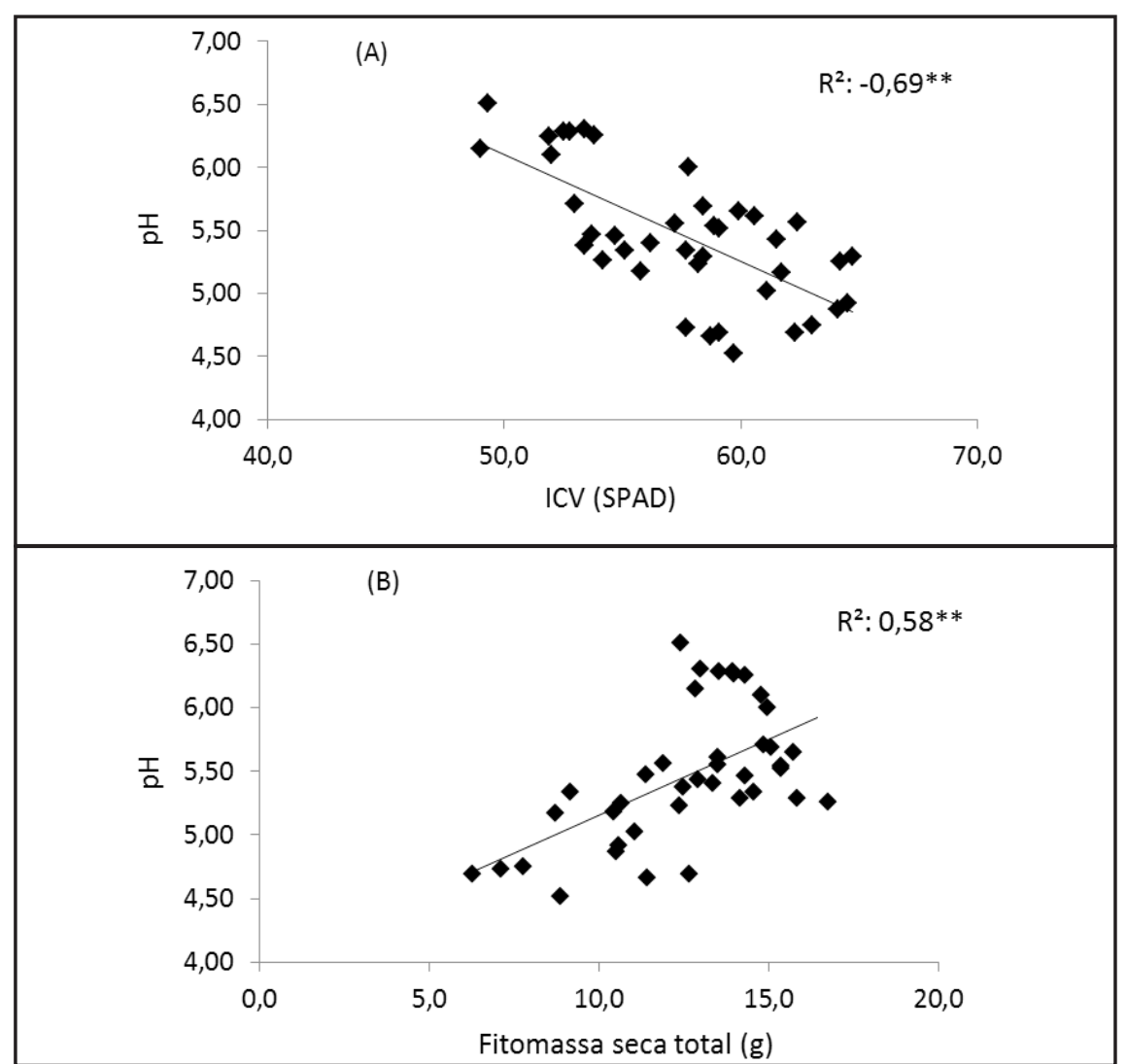

Figura 1. Intensidade de cor verde (ICV) em folhas novas completamente expandidas (A) e fitomassa seca da parte aérea (B) de plantas de gérbera e suas relações com o pH da solução drenada do substrato, aos 50 DAA (intensity of green color (ICV) in new fully expanded leaves (A) and dry matter of shoot (B) of gerbera plants and their relationship to the $\mathrm{pH}$ of the solution drained from de substrate, at 50 DAA). Botucatu, UNESP. 2008.

Guerrero (2009) obteve valores de 35 $\mathrm{mg} \mathrm{kg}^{-1}$ para cv. Red, podendo-se inferir que esses teores são adequados para essas cultivares. Teores inferiores a $25 \mathrm{mg}$ $\mathrm{kg}^{-1}$ são considerados insuficientes para gérbera, segundo Anjaneyulu (2008).

As características do substrato, em especial o $\mathrm{pH}$, influenciam a absorção de nutrientes e a produção de gérbera de vaso, podendo alterar a qualidade final da planta.

\section{REFERÊNCIAS}

ALBANO JP; MILLER WB; HALBROOKS MC. 1996. Iron toxicity stress causes bronze speckle, a specific physiological disorder of marigold (Tagetes erecta). Journal of the American Society for Horticultural Science 121: 430-437.

ANJANEYULU K. 2008. Diagnostic leaf nutrient norms and identification of yield limiting nutrients in gerbera grown under protected condictions using DRIS. Indian Journal of Horticulture 65: 176-179.

BARBOSA JG; BARBOSA MS; MUNIZ MA; CROSSI JAS. 2009. Nutrição mineral e adubação de plantas ornamentais. Informe Agropecuário 30: 16-21.
BRASIL. Instrução Normativa n.17, de 21 de maio de 2007. Aprova os Métodos Analíticos Oficiais para Análise de Substratos e Condicionadores de Solos. Diário Oficial da União, Brasília, 24 maio. Seção 1, p.8.

BUNT AC. 1984. Physical properties of mixtures of peats and minerals of different particle size and bulk density for potting substrates. Acta Horticulturae 150: 143-153.

CABALLERO R; ORDOVÁS J; PAJUELO P; CARMONA E; DELGADO A. 2007. Iron chlorosis in gerbera as related to properties of various types of compost used as growing Plant Analysis 38: 2357-2369.

CABALLERO R; PAJUELO P; ORDOVÁS J; CARMONA E; DELGADO A. 2009. Evaluation and correction of nutrient availability to Gerbera jamesonii in various compost-based growing media. Scientia Horticulturae 122: 244-250.

CAVINS TJ; WHIPKER BE; FONTENO WC; HARDEN B; McCALL I; GIBSON JL. 2000. Monitoring and managing $\mathrm{pH}$ and EC using the PourThru extraction method. Raleigh: Horticulture Information. 590: 1-17.

DE BOODT M; VERDONCK O. 1972. The physical properties of the substrates in horticulture. Acta Horticulturae 1: 37-44.

FERMINO MH. 1996. Aproveitamento de resíduos industriais e agricolas como alternativas de substratos hortícolas. Porto Alegre: UFRGS. media. Communications in Soil Science and 95p. (Tese mestrado).

FERMINO MH. 2002. O uso da analise física na avaliação da qualidade de componentes e substratos. In: FURLANI AMC; BATAGLIA OC; ABREU MF; ABREU CA; FURLANI PR; QUAGGIO JA; MINAMI K (coords). Caracterização, manejo e qualidade de substratos para produção de plantas. Documentos IAC, 70. Campinas: Instituto Agronômico. p.29-37.

GUERRERO AC. 2009. Aplicação de cloreto e silicato de potássio em gérbera (Gerbera jamesonii) de vaso. Botucatu: UNESP. 80p. (Tese mestrado).

GUERRERO AC; FERNANDES DM; LUDWIG F. 2012. Acúmulo de nutrientes em gérbera de vaso em função de fontes e doses de potássio. Horticultura Brasileira 30: 201-208.

HUMPHRIES JM; STANGOULIS JCR; GRAHAM RD. 2007. Manganese. In: BARKER AV; PIELBEAM DJ (coords). Handbook of Plant Nutrition. CRC Press. Boca Raton, p. 351-374.

JEONG KY; WHIPKER B; MCCALL I; GUNTER C; FRANTZ J. 2009. Characterization of nutrient disorders of gerbera hybrid 'Festival Light Eye Pink'. Acta Horticulturae 843: 177-182.

JONES JUNIOR JB; WOLF B; MILLS HA. 1996. Plant analysis handbook: a pratical sampling, preparation, analysis and interpretation guide? Athens: Micro-Macro Publishing. 213p.

LIN WC; FRENCH CJ. 1985. Effects of supplementary lighting and soil warming on flowering of three gerbera cultivars. HortScience 20: 271-273.

LUDWIG F. 2007. Cultivares de gérbera (Gerbera jamesonii), em vaso, sob dois niveis de fertirrigação. Botucatu: UNESP. 79p. (Tese mestrado).

LUDWIG F; FERNANDES DM; MOTA PRD; VILLAS BÔAS RL. 2008. Macronutrientes em cultivares de gérbera sob dois níveis de fertirrigação. Horticultura Brasileira 26: 68-73.

LUDWIG F; GUERRERO AC; FERNANDES DM; VILLAS BÔAS RL. 2010. Análise de crescimento de gérbera de vaso conduzida em diferentes substratos. Horticultura Brasileira 28: 70-74.

MALAVOLTA E; VITTI GC; OLIVEIRA SA. 1997. Avaliação do estado nutricional das plantas: princípios e aplicações. Piracicaba: Potafos. 319p.

MERCURIO G. 2002. Gerbera cultivation in greenhouse. The Netherlands: Schreurs. 206p.

MERHAUT DJ. 2007. Magnesium. In: BARKER AV; PIELBEAM DJ (coords). Handbook of Plant Nutrition. CRC Press. Boca Raton, p. 145-182.

RAIJ BV; ANDRADE JC; CANTARELLA H; QUAGGIO JA. 2001. Análise química da fertilidade dos solos tropicais. Campinas: IAC. $285 \mathrm{p}$.

ROGERS MN; TJIA BOS. 1990. Gerbera production for cut flowers and pot plants. Portland: Timber Press. 116p.

SONNEVELD C; ELDEREN CW, 1994 Chemical analysis of peaty growing media by means of water extraction. Communications on Soil Science and Plant Analysis 25: 31993208.

SONNEVELD C; VOOGT W. 1997. Effects of pH value and $\mathrm{Mn}$ application on yield and nutrient absorption with rockwool grown gerbera (refereed). Acta Horticulturae 450: 139-147. 